\title{
Vascularized Tissue to Reduce Fistula Following Salvage Total Laryngectomy: A Systematic Review
}

Vinidh Paleri, MS, FRCS (ORL-HNS); Mike Drinnan, PhD; Michiel W. M. van den Brekel, MD, PhD; Michael L. Hinni, MD; Patrick J. Bradley, MB, BCh, BAO; Gregory T. Wolf, MD; Remco de Bree, MD, PhD; Johannes J. Fagan, MBChB, MMed; Marc Hamoir, MD; Primož Strojan, MD; Juan P. Rodrigo, MD; Kerry D. Olsen, MD; Phillip K. Pellitteri, DO, FACS; Ashok R. Shaha, MD; Eric M. Genden, MD; Carl E. Silver, MD, FACS; Carlos Suárez, MD; Robert P. Takes, MD, PhD; Alessandra Rinaldo, MD, FACS, FRCSGlasg; Alfio Ferlito, MD, FACS, FHKCORL

\begin{abstract}
Objectives/Hypothesis: Pharyngocutaneous fistulae (PCF) are known to occur in nearly one-third of patients after salvage total laryngectomy (STL). PCF has severe impact on duration of admission and costs and quality of life and can even cause severe complications such as bleeding, infection and death. Many patients need further surgical procedures. The implications for functional outcome and survival are less clear. Several studies have shown that using vascularized tissue from outside the radiation field reduces the risk of PCFs following STL. This review and meta-analysis aims to identify the evidence base to support this hypothesis.

Data Sources: English language literature from 2004 to 2013

Review Methods: We searched the English language literature for articles published on the subject from 2004 to 2013.

Results: Adequate data was available to identify pooled incidence rates from seven articles. The pooled relative risk derived from 591 patients was 0.63 (95\% CI: 0.47 to 0.85 ), indicating that patients who have flap reconstruction/reinforcement reduced their risk of PCF by one-third.

Conclusion: This pooled analysis suggests that there is a clear advantage in using vascularized tissue from outside the radiation field in the laryngectomy defect. While some studies show a clear reduction in PCF rates, others suggest that the fistulae that occur are smaller and rarely need repair.

Key Words: Salvage laryngectomy; pharyngocutaneous fistula; free flaps; pedicled flaps; reconstruction; recurrent laryngeal cancer.
\end{abstract}

Laryngoscope, 124:1848-1853, 2014

\section{INTRODUCTION}

The Veteran's Affairs Cooperative Laryngeal Cancer Study Group and Radiation Therapy Oncology Group (RTOG)-91-11 trials $^{1,2}$ showed the efficacy of chemora-

From the Department of Otolaryngology-Head and Neck Surgery (v.P., M.D.), Newcastle upon Tyne Foundation Hospitals NHS Trust, Newcastle upon Tyne, the Department of Otolaryngology-Head and Neck Surgery (P.J.B.), Nottingham University Hospital, Queens Medical Centre, Nottingham, U.K; the Department of Head and Neck Surgery and Oncology (M.w.M. vDB.), Netherlands Cancer Institute-Antoni van Leeuwenhoek Hospital, Department of Otolaryngology-Head and Neck Surgery (R.DB.), VU University Medical Center, Amsterdam; the Department of Otolaryngology-Head and Neck Surgery (R.P.T.), Radboud University Medical Center, Nijmegen, The Netherlands; the Department of Otolaryngology-Head and Neck Surgery (M.L.H.), Mayo Clinic, Phoenix, Arizona; the Department of Otolaryngology-Head and Neck Surgery, University of Michigan (G.T.w.), Ann Arbor, Michigan; the Department of Otorhinolaryngology (к.D.o.), Mayo Clinic, Rochester, Minnesota; the Department of Otolaryngology-Head and Neck Surgery (P.K.P.), Guthrie Health System, Sayre, Pennsylvania; the Head and Neck Service (A.R.s.), Memorial Sloan-Kettering Cancer Center, New York; the Department of Otolaryngology-Head and Neck Surgery (E.M.G.), The Mount Sinai Medical Center, New York; the Division Departments of Surgery and Otolaryngology-Head and Neck Surgery (c.E.s.), Albert Einstein College of Medicine, Montefiore Medical Center, Bronx, New York, U.S.A; the diation (CRT) in the management of locally advanced tumors of the larynx. With primary CRT being widely adopted as a management strategy, there has been a paradigm shift in the treatment of laryngeal cancer.

Departments of Otolaryngology, Faculty of Health Sciences (J.J.F.), University of Cape Town, Cape Town, South Africa; the Department of Head and Neck Surgery, Head and Neck Oncology Program (м.н.), St Luc University Hospital and Cancer Center, Brussels, Belgium; the Department of Radiation Oncology (P.S.), Institute of Oncology, Ljubljana, Slovenia; the Department of Otolaryngology (J.P.R., C.S.), Hospital Universitario Central de Asturias; the Instituto Universitario de Oncología del Principado de Asturias (J.P.R., C.s.), Oviedo, Spain; the ENT Clinic (A.R., A.F.), University of Udine, Udine, Italy

Presented in part at the Head and Neck Special Interest Group (North) Annual meeting, Royal College of Speech and Language Therapists, Newcastle upon Tyne, UK, January 16, 2013.

The authors have no funding, financial relationships, or conflicts of interest to disclose.

Send correspondence to Vinidh Paleri, MS, FRCS (ORL-HNS), Department of Otolaryngology-Head and Neck Surgery, Newcastle upon Tyne Foundation Hospitals NHS Trust, Newcastle upon Tyne, U.K. E-mail: vinidh.paleri@ncl.ac.uk

DOI: 10.1002/lary.24619 
With organ preservation rates between $60 \%$ and $80 \%$ at 5 years for those patients completing the therapy, there continues to be a substantial proportion of patients who need salvage total laryngectomy (STL), commonly due to persistent or recurrent laryngeal cancer, second primary tumors, and occasionally for laryngeal dysfunction or radionecrosis. ${ }^{3}$ It is widely accepted that salvage total laryngectomies (STLs) performed after radiation failure are more prone to complications as compared to laryngectomies performed upfront. A higher complication rate is reported for patients who undergo STL with neck dissections and flap reconstructions of mucosal defects. The most significant complication in this regard is a pharyngocutaneous fistula (PCF). ${ }^{4}$ A PCF can have several unintended consequences. It delays postoperative recovery, delays function acquisition and increases hospital stay and consequently costs. Rarely, a salivary leak can erode into cervical or mediastinal blood vessels causing potentially catastrophic bleeding and life-threatening complications.

A meta-analysis of 25 studies by Paydarfar and Birkmeyer ${ }^{5}$ demonstrated a 2.28 times increased risk of PCF among patients who received radiation or CRT prior to laryngectomy. Weber et al. ${ }^{6}$ published the outcomes of 129 patients in the RTOG 91-11 trial who needed STL. The incidence of PCF in patients who underwent CRT was $30 \%$, which was twice that seen in patients who failed radiation therapy alone. Additionally, the incidence of complications in the $30 \%$ of patients who need salvage laryngectomy for failed CRT can be as high as $50 \%$ to $60 \% .^{6-8}$ Complications are more common when STLs are performed within the first year after CRT. ${ }^{9}$ Several reports suggest that PCFs following CRT are more likely to need surgical repair than are those that occur after primary laryngectomy. ${ }^{5,7,10}$

Not all studies report a higher complication rate for STLs. This may be a reflection of the data analysis and the variable treatment regimens employed, as well as the year in which the laryngectomy was performed. For instance, Grau et al. ${ }^{11}$ studied the incidence of fistula following laryngectomy in radiation failures in the Danish Head and Neck Cancer Group (DAHANCA) trials (5, 6, and 7) over a 10-year period between 1987 and 1997. They found a $19 \%$ incidence of PCF in 472 patients. Multiple logistic regression analysis showed that STLs performed in the latter years of the 10-year period, advanced $\mathrm{T}$ stage and nonglottic location of primary sites predisposed to PCF occurrence. Given the intensified (accelerated) radiotherapy schedules in the DAHANCA 6 and 7 trials, the higher PCF incidence may reflect more aggressive irradiation schedules in the latter years. However, a higher proportion of advanced tumors undergoing STL and a reduction in the number of STLs over the 10-year period (i.e., as few as 30 STLs performed at five different centers in 1997) may also have contributed to the observed increase in relative risk of fistula.

Radiotherapy and CRT not only kill tumor cells, but also cause damage to the other tissues, including blood vessels. $^{12}$ This tissue damage causes delayed healing
TABLE I.

Inclusion Criteria.

1. The cohort should include salvage laryngectomy patients who underwent some form of flap reconstruction to the neopharynx, whose outcomes should be distinctly identifiable.

2. The number patients who develop pharyngocutaneous fistula following salvage laryngectomy and flap reconstruction should be clearly identified.

3. A clear description of flap used to reconstruct the neopharynx should be available.

after radiotherapy and CRT. Several surgical teams have hypothesized that transferring healthy vascularized tissue from outside the radiation field enhances wound healing and reduces the incidence of wound complications. Several studies have reported on the use of vascularized tissue from outside the radiation field to reinforce the pharyngeal repair site or to augment the circumference by using this as a patch. The choices of donor tissue are usually: pectoralis major myofascial flap (PMMF), pectoralis major myocutaneous flap (PMMC), or free flaps from various sites. While some have been supportive of this intervention, others have not identified an advantage. The outcome of interest, the $\mathrm{PCF}$, is not subject to a diagnostic bias and is a clean binary outcome (presence or not of a salivary leak).

The aim of this study was to pool the available data on PCF rates in patients after STL who received flap cover following laryngectomy, and to quantify any benefit associated with this strategy.

\section{MATERIALS AND METHODS}

\section{Systematic Review}

CRT as a strategy for advanced laryngeal cancer has been used since the publication of the RTOG 91-11 trial, ${ }^{2}$ and general awareness of the increased risk of PCFs following STLs from the RTOG 91-11 cohort has been available following the publication by Weber et al. ${ }^{6}$ Both these reports were published in 2003; therefore, the English language literature from 2004 to 2013 was searched by a librarian with relevant expertise. The following search terms were used: salvage total laryngectomy, pharyngocutaneous fistula, salivary fistula, salivary leak, complications, flap reconstruction, free flap, and pedicled flap. International experts were also consulted. Inclusion criteria are set out in Table I.

\section{Statistical Analysis}

Data were tabulated in Microsoft Excel, then exported for analysis using the statistical package $R$ Version 3.0 (The $R$ Foundation for Statistical Computing, Vienna, Austria).

To measure heterogeneity of the incidence data, the $\mathrm{I}^{2}$ statistic was calculated, and the data were visualized using a funnel plot. A high figure for $\mathrm{I}^{2}$ implies heterogeneous data with the converse implying homogenous data. Because the data ranged from entirely homogenous to moderately heterogeneous, we used an inverse variance (fixed effects) model to calculate the pooled estimates of PCF rates. Similarly, the fixed effects model of Mantel-Haenszel was used to calculate pooled estimates of relative risk. This is the probability of fistula in the reconstruction group, divided by the probability of the event in the primary repair group. 
TABLE II.

Summary Data From the included Studies.

\begin{tabular}{|c|c|c|c|c|c|c|c|c|}
\hline & & & $\mathbf{N}$ & PCF & N & PCF & N & PCF \\
\hline Author & Type of Flap & Reconstruction & Total & Total & Primary & Primary & Flap & Flap \\
\hline Fung et al. $^{21}$ & Free & reinforcement & 41 & 12 & 27 & 8 & 14 & 4 \\
\hline Gil et al. ${ }^{10}$ & PMMF & reinforcement & 80 & 19 & 69 & 16 & 11 & 3 \\
\hline Oosthuizen et al. ${ }^{27}$ & PMMF & reinforcement & 10 & 3 & 2 & 1 & 8 & 2 \\
\hline Patel et al. ${ }^{18}$ & Free \& PMMF & reinforcement \& patch & 359 & 94 & 99 & 34 & 260 & 60 \\
\hline Powell et al. ${ }^{19}$ & Free \& PMMF & reinforcement \& patch & 45 & 10 & 38 & 10 & 7 & 0 \\
\hline Righini et al. ${ }^{20}$ & PMMF & reinforcement & 25 & 5 & 12 & 4 & 13 & 1 \\
\hline Sousa et al. ${ }^{22}$ & PMMC & not specified & 31 & 10 & 12 & 7 & 19 & 3 \\
\hline${ }^{*}$ Patel and Keni ${ }^{17}$ & PMMF & reinforcement & 17 & 4 & 7 & 4 & 10 & 0 \\
\hline${ }^{*}$ Withrow et al. ${ }^{16}$ & Free & patch & 37 & 13 & 20 & 10 & 17 & 3 \\
\hline Summary & & & 591 & 153 & 259 & 80 & 332 & 73 \\
\hline
\end{tabular}

*Shown for completeness only; most patients are also reported in Patel et al. ${ }^{18}$ Data are not included in the summary statistics or meta-analysis.

$\mathrm{PCF}$ = pharyngocutaneous fistulae; PMMC = pectoralis major myocutaneous flap; PMMF = pectoralis major myofascial flap.

\section{RESULTS}

\section{Systematic Review}

The Medline search identified 71 abstracts. Full text was obtained wherever the abstract indicated that vascularized tissue had been used to reconstruct the neopharynx. After screening against the inclusion criteria, 12 articles were retained. Finally, after further exclusions as described below, seven articles were included in the final meta-analysis. Table II summarizes this literature on using new vascularized tissue to reconstruct neopharyngeal defects. The commentary below expands on some of the key findings.

\section{Exclusions}

Two $\operatorname{articles}^{13,14}$ were excluded from the analysis because it was impossible to separate the data pertaining to laryngectomy alone from those who underwent more complex resections. Data from three other papers ${ }^{15-17}$ despite being eligible, were also excluded for the reasons stated below:

1. Sakai et al. ${ }^{13}$ described their experience of bringing in vascularized tissue in 13 of 52 patients undergoing a STL or a salvage total laryngopharyngectomy. PMMC or deltopectoral flaps were used to reinforce the pharyngeal repair; or in patients undergoing total laryngopharyngectomy, these flaps were used to cover the jejunal free flap. Despite more patients in the flap group receiving primary CRT than radiation alone (53.8\% vs. $30.8 \%$ ), the PCF rate was lower in the flap group (1 patient, $7.7 \%$ ) compared with the PCF rate in the nonflap group (12 patients, $30.1 \%$ ). However, we were unable to separate out the data pertaining to STL patients in this series.

2. Sinclair et $\mathrm{al}^{14}$ summarized their experience with 137 patients who underwent laryngectomy or laryngopharyngectomy with free flap reconstruction of the pharyngeal defect. Most patients had a patch graft to augment the circumference. The major aim of this article was to identify whether performing a primary tracheoesophageal puncture increased PCF rates; however, it can be gleaned from the article that, despite a complex cohort including 32 laryngopharyngectomies, the incidence of PCF in this large group was $14 \%$. In their discussion of results, Sinclair et al. ${ }^{14}$ are of the view that it is the use of vascularized free flaps that has helped to keep the rates of PCFs low in this cohort, despite the vast majority being STLs. This article was excluded because both primary and salvage patients belonged to the cohort, and the data for salvage patients was not separately presented.

3. Although adequate data was present in another article, ${ }^{15}$ it was excluded as it was determined that the patient cohort was different from the other series: All patients had extensive tumors fungating through the skin and were malnourishedand seven cases also underwent partial pharyngeal resection.

4. Finally, there was overlap between patients reported in two eligible articles ${ }^{16,17}$ and a recent multi-institutional review ${ }^{18}$ of 359 patients from seven centers. Assuming an even recruitment, approximately 40 patients would have been represented twice in the meta-analysis, receiving double the weighting of all others. Therefore, we conducted our metaanalysis without the two smaller studies, although we have reported their data for completeness.

\section{Commentary on Surgical Methods and Outcomes}

In broad terms, surgeons have adopted two distinct techniques when using vascularized tissue following laryngectomy:

A.Vascularized tissue to augment the pharyngeal circumference by a patch graft. Withrow et al. ${ }^{16}$ compared two groups of STL patients: Seventeen patients had free flaps to augment the pharyngeal circumference and 20 patients underwent primary closure of the pharynx. The cutaneous paddles of the free flaps were placed as patch grafts to supplement the anterior pharyngeal defects following laryngectomy. This free flap reconstruction group had a lower rate of fistula (18\%) compared with the primary closure group (50\%).

Powell et al. ${ }^{19}$ detailed the course of 45 STLs: Thirty-eight STLs 38 underwent primary closure and seven STLs had free or pedicled flaps employed as a patch to augment the pharyngeal closure.There were no leaks in the group that had a flap, whereas a $26 \%$ leak rate was evident in the primary closure group. 
TABLE III.

Proportions of Pharyngocutaneous Fistula by Group, With Relative Risk of Fistula Between the Groups.

\begin{tabular}{|c|c|c|c|c|}
\hline & $\%$ PCF & $\%$ PCF & $\%$ PCF & Relative Risk \\
\hline Author & Overall & Primary & Flap & Flap Versus Primary \\
\hline Fung et al. ${ }^{21}$ & $29.3(16.1-45.5)$ & 29.6 (13.8-50.2) & $28.6(8.4-58.1)$ & $0.96(0.35-2.65)$ \\
\hline Gil et al. ${ }^{10}$ & $23.8(14.9-34.6)$ & 23.1 (13.9-34.9) & $27.2(6.0-61.0)$ & $1.18(0.41-3.38)$ \\
\hline Oosthuizen et al. ${ }^{27}$ & $30.0(6.7-65.2)$ & $50.0(1.3-98.7)$ & $25.0(3.2-65.1)$ & $0.50(0.08-3.13)$ \\
\hline Patel et al. ${ }^{18}$ & $26.2(21.7-31.1)$ & $34.3(25.1-44.6)$ & 23.1 (18.1-28.7) & $0.67(0.47-0.95)$ \\
\hline Powell et al. ${ }^{19}$ & $22.2(11.2-37.1)$ & $26.3(13.4-43.1)$ & $0.0(0.0-41.0)$ & $0.24(0.02-3.74)$ \\
\hline Righini et al. ${ }^{20}$ & $20.0(6.8-40.7)$ & $33.3(9.9-65.1)$ & $7.7(0.2-36.0)$ & $0.23(0.03-1.79)$ \\
\hline Sousa et al. ${ }^{22}$ & $32.3(16.7-51.4)$ & $58.3(27.7-84.8)$ & $15.8(3.4-39.6)$ & $0.27(0.09-0.85)$ \\
\hline${ }^{*}$ Patel and Keni ${ }^{17}$ & $23.5(6.8-49.8)$ & $57.1(18.4-90.1)$ & $0.0(0.0-30.8)$ & $0.08(0.00-1.26)$ \\
\hline${ }^{*}$ Withrow et al. ${ }^{16}$ & $35.1(20.2-52.5)$ & $50.0(27.2-72.8)$ & $17.6(3.8-43.4)$ & $0.35(0.12-1.08)$ \\
\hline Summary & 26.1 (22.7-29.7) & $31.2(25.8-36.9)$ & 22.2 (17.9-26.8) & $0.63(0.47-0.85)$ \\
\hline
\end{tabular}

PCF = pharyngocutaneous fistulae.

$95 \%$ confidence Intervals are in brackets.

Test for heterogeneity on relative risk: $X^{2}(6)=5.7(P$-value $=0.5)$. No evidence for heterogeneity, thus fixed effects model used

Summary proportions calculated using inverse variance method.

Summary relative risk calculated using Mantel-Haenszel method.

${ }^{\star}$ Shown for completeness only; most patients are also reported in Patel et al. ${ }^{18}$ Data are not included in the summary statistics or meta-analysis.

B.Vascularized tissue to reinforce the pharyngeal repair. The use of PMMF to reinforce the defect is well described by Righini et al, ${ }^{20}$ who used the PMMF flap to reinforce the suture line by covering it with healthy muscle, anchored to the base of tongue, the pharyngeal constrictors, and the posterior wall of the trachea. This led to a $7.6 \%$ leak rate compared to $33.3 \%$ $\mathrm{PCF}$ rates in those who received no flaps. Other investigators have used similar techniques. Patel et al. ${ }^{17}$ reported on a series of 17 patients who underwent STL: Seven patients were closed primarily and 10 patients had reinforcement of the repair line with PMMF. Although none of the patients in the PMMF group developed PCF, four of seven patients who were closed without reinforcement developed fistula $(P<0.02)$.

Gil et al. ${ }^{10}$ assessed the role of PMMF in 80 patients who underwent STL. Of those, 69 patients were closed primarily and 11 patients received a PMMF reinforcement of the suture line. Whereas there was no difference in the rates of PCF formation between the groups (27\% vs. $24 \%$ ), eight of the 16 patients (50\%) with PCF who did not receive PMMF required a second surgical procedure for fistula closure. On the other hand, none of the patients in the PMMF group who developed a PCF required a second operation $(P<.001)$, or had a hospital stay exceeding 30 days.

Fung et al. ${ }^{21}$ retrospectively compared a cohort of 14 patients who had free tissue reinforcement of the pharyngeal suture line following STL after CRT with a historical group of 27 patients (surgical patients of RTOG 91-11 trial) who had primary repair alone. Although the overall PCF rate was similar between groups (29\% in the flap group compared with $30 \%$ in the RTOG-91-11 group) the RTOG 91-11 group had a 15\% incidence of major wound complications, while the flap group had none. All PCFs in the flap group were minor and needed no surgical repair, while the three in the primary repair group were considered to be major. All patients underwent primary tracheoesophageal punc- ture. The results of this study raise the question: Would avoiding a primary puncture in these patients have made a difference to the fistula rates?

Whereas most studies have specified how exactly the flap was used to help with the neopharyngeal reconstruction, Sousa et al. ${ }^{22}$ did not describe how the PMMC flap was inset in 19 of 31 STLs performed at their center. However, outcome data and flap use are well described, and as this article satisfied inclusion criteria, it was included.

\section{Pooled Estimates of Incidence}

Table III summarizes the overall incidence of PCF in all included studies and by the two surgical groups. From all 591 patients in the seven studies, the pooled (95\% confidence interval) fistula rate was $26.1 \%$ (22.7 to $29.7 \%$ ). The pooled incidence for 332 patients who underwent flap reconstruction was $22.2 \%$ (17.9 to $26.8 \%$ ), but was $31.2 \%$ (25.8 to 36.9 ) in 259 patients who were closed primarily (Table III). Based on the event rates in both groups, we estimate that the number needed to treat is 11 ; this means that to prevent one fistula, 11 patients will need vascularized flaps following STL.

\section{Pooled Estimates Of Relative Risk}

The pooled relative risk for patients who have flap reconstruction/reinforcement was 0.63 (95\% CI: 0.47 to 0.85 ), relative to those whose pharyngeal defects are closed primarily (Fig. 1). In other words, the risk of fistula following primary closure is approximately 50\% higher than for the flap reconstruction.

\section{DISCUSSION}

\section{Summary of Main Results}

This article lends support to the hypothesis that employing vascularized tissue from outside the radiation 


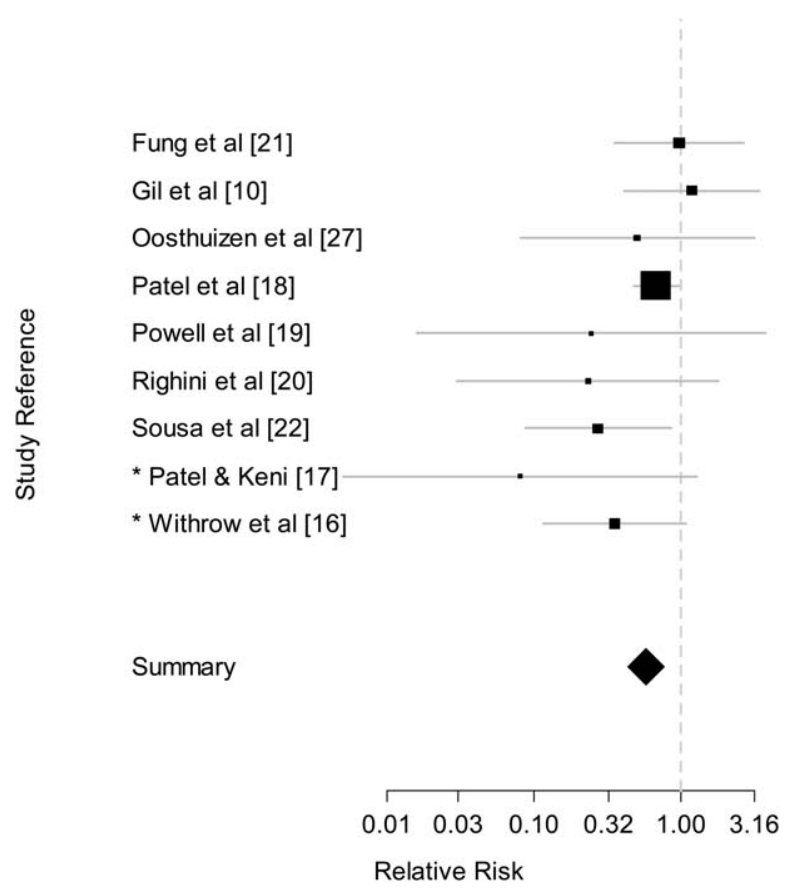

Fig. 1. Forest plot for relative risk of developing pharyngocutaneous fistula. The pooled relative risk for those patients who receive flap reconstruction/reinforcement of the neopharynx is $0.63(95 \%$ confidence interval: $0.47-0.85$ ) *Shown for completeness only; most patients are also reported in Patel et al. ${ }^{18}$ Data are not included in the summary statistics or meta-analysis.

field appears to be consistently effective in reducing the incidence of postoperative PCFs in a third of patients who undergo STL and is a significant advantage in this patient group.

\section{Quality of Evidence and Potential Biases in Review}

We appreciate that this is a retrospective pooling of case series and thus is prone to the drawbacks associated with such studies. However, the presence or absence of PCF is a binary outcome that is relatively immune to measurement bias and therefore should be robust.

\section{Comparison With Other Reviews}

Patel et al. ${ }^{18}$ performed a multi-institutional retrospective review of 359 STL patients, grouped by closure technique: primary closure of the defect $(n=99)$, interposed free tissue $(n=220)$ or PMMF onlay flap $(n=40)$. Incidence of PCF was $34 \%, 25 \%$ and $15 \%$ for the primary closure, interposed free tissue and PMMF onlay groups respectively, with the difference in fistula duration between primary closure and either flap technique being statistically significant $(p=.004)$. For patients who developed fistula, mean duration of fistula was significantly prolonged with primary closure (14.0 weeks) compared with pectoralis flap (9.0 weeks) and free flap (6.5 weeks). Since this is the single biggest contributor to our own systematic review, it is no surprise that the two reviews are in close agreement about the overall benefit of flap techniques. However, our review captures the international practice on this subject and confirms that similar reductions in PCF rates are achieved (although not achieving statistical significance owing to small sample sizes) in all the studies except one.

In patients undergoing primary laryngectomy, Smith et al. ${ }^{23}$ demonstrated a significant reduction in the incidence of PCF by the routine addition of a PMMF to reinforce the pharyngeal closure in a retrospective cohort of 223 consecutive primary total laryngectomies, from $23 \%$ to less than $1 \%$. Similarly, in a cohort of 65 patients, Albirmawy ${ }^{24}$ used a sternocleidomastoid muscle collar flap and reduced the PCF rates from $34 \%$ to $3 \%$. Although the use of flaps ${ }^{25,26}$ is associated with donor site morbidity, it can be anticipated that this morbidity outweighed by the significant morbidity and risks associated with PCF development.

\section{Implications for Clinical Practice}

This strategy is simple and well within the expertise of the majority of head and neck surgical teams anywhere in the world. Based on the studies presented, it is possible to reduce PCF rates following STL to well below those seen even after primary laryngectomy. The increased time spent in the operating suite to perform a flap reconstruction may be offset by the reduction in postoperative stay and reduced need for surgical repair of the fistula. Other outcomes such as the need for a second surgical procedure to close the PCF, donor site morbidity, extra costs of the flap reconstruction and a description of the fistula as major or minor were not analyzed as the clinical assessment of the size of the fistula can vary, as can the decision to do a second operation. However, it should be noted that several studies ${ }^{10,13,20,21}$ indicated that surgical repair of fistulae was almost never required in patients who received prophylactic flap reconstruction.

It is very unlikely that an adequately powered trial to answer this question will ever be performed, and clinicians will have to make the best use of available data to change practice. As the effects of the complication are severe in this vulnerable group of patients, all the authors (despite their geographical distribution) felt that the heterogeneity in technique was not clinically significant, the overall effect is the same and were comfortable pooling data. Given the substantial reduction in fistula rates, the benefit conferred by a vascularized tissue reconstruction is strong. A multi-site, multi-author review such as this has a powerful role to play in changing practice in the absence of high level evidence.

\section{Implications for Research}

Definitive answers concerning the functional outcome and cost-effectiveness of routine flap reconstruction in STL would require further study.

\section{CONCLUSION}

There is currently retrospective, but clear evidence to support the routine use of vascularized flaps to 
augment or bolster the pharyngeal closure following STL as a strategy to reduce PCF. The risk of PCF in these patients can be reduced by one-third if vascularized tissue from outside the radiation field is used to reconstruct the laryngectomy defect. The vascularized tissue can be used to reinforce the pharyngeal repair site or to augment the circumference of the neopharynx by using it as a patch. While it is appreciated that flaprelated morbidity exists, the morbidity associated with a PCF is undoubtedly greater, thus supporting the aphorism that "prevention is better than any subsequent management and treatment of PCF."

\section{Acknowledgement}

This article was written by members and invitees of the International Head and Neck Scientific Group (www. IHNSG.com).

\section{BIBLIOGRAPHY}

1. The Department of Veterans Affairs Laryngeal Cancer Study Group Induction chemotherapy plus radiation compared with surgery plus radiation in patients with advanced laryngeal cancer. $N$ Engl J Med 1991;324:1685-1690.

2. Forastiere AA, Goepfert H, Maor M, et al. Concurrent chemotherapy and radiotherapy for organ preservation in advanced laryngeal cancer. $N$ Engl J Med 2003;349:2091-2098.

3. Theunissen EA, Timmermans AJ, Zuur CL, et al. Total laryngectomy for a dysfunctional larynx after (chemo)radiotherapy. Arch Otolaryngol Head Neck Surg 2012;138:548-555.

4. van der Putten L, de Bree R, Kuik DJ, et al. Salvage laryngectomy: oncological and functional outcome. Oral Oncol 2011;47:296-301.

5. Paydarfar JA, Birkmeyer NJ. Complications in head and neck surgery: a meta-analysis of postlaryngectomy pharyngocutaneous fistula. Arch Otolaryngol Head Neck Surg 2006;132:67-72.

6. Weber RS, Berkey BA, Forastiere A, et al. Outcome of salvage total laryngectomy following organ preservation therapy: the Radiation Therapy Oncology Group trial 91-11. Arch Otolaryngol Head Neck Surg 2003; 129:44-49.

7. Ganly I, Patel S, Matsuo J, et al. Postoperative complications of salvage total laryngectomy. Cancer 2005;103:2073-2081.

8. Sewnaik A, Keereweer S, Al-Mamgani A, et al. High complication risk of salvage surgery after chemoradiation failures. Acta Otolaryngol 2012, 132:96-100.

9. Sassler AM, Esclamado RM, Wolf GT. Surgery after organ preservation therapy. Analysis of wound complications. Arch Otolaryngol Head Neck Surg 1995;121:162-165.
10. Gil Z, Gupta A, Kummer B, et al. The role of pectoralis major muscle flap in salvage total laryngectomy. Arch Otolaryngol Head Neck Surg 2009; 135:1019-1023.

11. Grau C, Johansen LV, Hansen HS, et al. Salvage laryngectomy and pharyngocutaneous fistulae after primary radiotherapy for head and neck cancer: a national survey from DAHANCA. Head Neck 2003;25:711-716.

12. Russell NS, Hoving S, Heeneman S, et al. Novel insights into pathological changes in muscular arteries of radiotherapy patients. Radiother Oncol 2009;92:477-483.

13. Sakai A, Okami K, Sugimoto R, et al. Prevention of wound complications in salvage pharyngolaryngectomy by the use of well-vascularized flaps. Acta Otolaryngol 2012;132:778-782.

14. Sinclair CF, Rosenthal EL, McColloch NL, et al. Primary versus delayed tracheoesophageal puncture for laryngopharyngectomy with free flap reconstruction. Laryngoscope 2011;121:1436-1440.

15. Mebeed AH, Hussein HA, Saber TKh, Zohairy MA, Lotayef M. Role of pectoralis major myocutanuos flap in salvage laryngeal surgery for prophylaxis of pharyngocutaneuos fistula and reconstruction of skin defect. $J$ Egypt Natl Cancer Inst 2009;21:23-32.

16. Withrow KP, Rosenthal EL, Gourin CG, et al. Free tissue transfer to manage salvage laryngectomy defects after organ preservation failure. Laryngoscope 2007;117:781-784.

17. Patel UA, Keni SP. Pectoralis myofascial flap during salvage laryngectomy prevents pharyngocutaneous fistula. Otolaryngol Head Neck Surg 2009; 141:190-195.

18. Patel UA, Moore BA, Wax M, et al. Impact of pharyngeal closure technique on fistula after salvage laryngectomy. JAMA Otolaryngol Head Neck Surg 2013;139:1156-1162.

19. Powell J, Ullal U, Ahmed O, Ragbir M, Paleri V. Tissue transfer during salvage laryngectomy following chemoradiation to prevent pharyngocutaneous fistula. J Laryngol Otol 2014

20. Righini C, Lequeux T, Cuisnier O, Morel N, Reyt E. The pectoralis myofascial flap in pharyngolaryngeal surgery after radiotherapy. Eur Arch Otorhinolaryngol 2005:262:357-361.

21. Fung K, Teknos TN, Vandenberg CD, et al. Prevention of wound complications following salvage laryngectomy using free vascularized tissue. Head Neck 2007;29:425-430.

22. Sousa AA, Castro SM, Porcaro-Salles JM, et al. The usefulness of a pectoralis major myocutaneous flap in preventing salivary fistulae after salvage total laryngectomy. Braz J Otorhinolaryngol 2012;78:103-107.

23. Smith TJ, Burrage KJ, Ganguly P, Kirby S, Drover C. Prevention of postlaryngectomy pharyngocutaneous fistula: the Memorial University experience. J Otolaryngol 2003;32:222-225.

24. Albirmawy OA. Prevention of postlaryngectomy pharyngocutaneous fistula using a sternocleidomastoid muscle collar flap. J Laryngol Otol 2007; 121:253-257.

25. Merve A, Mitra I, Swindell R, Homer JJ. Shoulder morbidity after pectoralis major flap reconstruction for head and neck cancer. Head Neck 2009; 31:1470-1476.

26. de Witt CA, de Bree R, Verdonck-de Leeuw IM, Quak JJ, Leemans CR. Donor site morbidity of the fasciocutaneous radial forearm flap: what does the patient really bother? Eur Arch Otorhinolaryngol 2007;264: 929-934.

27. Oosthuizen JC, Leonard DS, Kinsella JB. The role of pectoralis major myofascial flap in salvage laryngectomy: a single surgeon experience. Acta Otolaryngol 2012;132:1002-1005. 